\title{
Prostatic Hemorrhage, CTCAE
}

National Cancer Institute

\section{Source}

National Cancer Institute. Prostatic Hemorrhage, CT CAE. NCI Thesaurus. Code C56552.

A disorder characterized by bleeding from the prostate gland. 